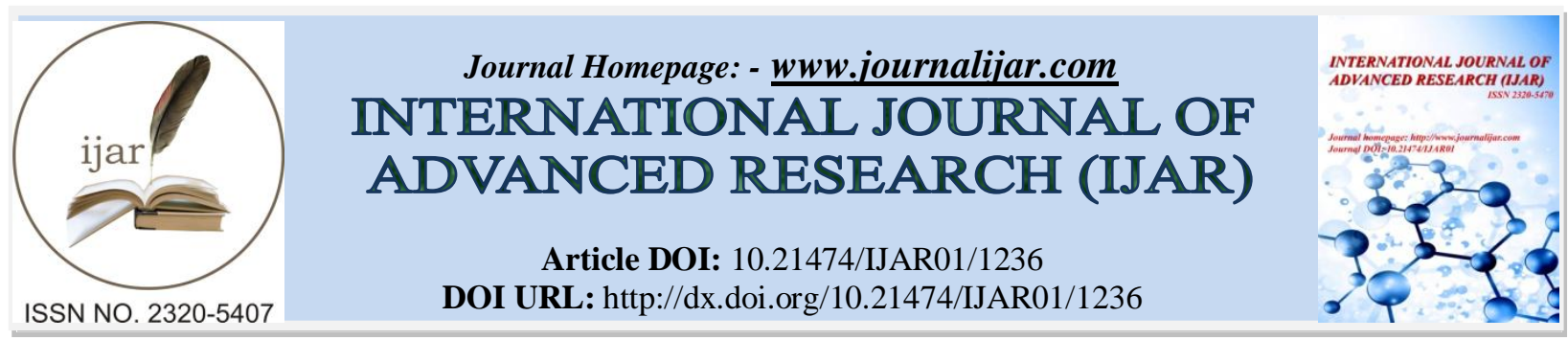

RESEARCH ARTICLE

\title{
TAXONOMY AND SEEDLING MORPHOLOGY OF SIX SPECIES OF DIGITARIA (POACEAE) FROM VINDHYAN REGION, UTTAR PRADESH, INDIA.
}

Ajai Kumar Singh, Ayush Kumar Singh and Manish Kumar Srivastava.

Department of Botany, Udai Pratap College (Autonomous), Varanasi-221 002, India.

\section{Manuscript Info}

Manuscript History

Received: 18 June 2016

Final Accepted: 20 July 2016

Published: August 2016

Key words:-

Grass, Seedling.

\begin{abstract}
Present paper incorporates the taxonomic study of six species of genus Digitaria Heist. Ez. Fab., family Poaceae, viz. D. abludens, D. ciliaris, $D$. griffithii, D. longiflora, D. ternate and D. violascens, collected from Vindhyan Region of Uttar Pradesh, India. Seedling morphology of reported taxa have also been recorded. Taxonomic key to the mature plants and seedlings have been prepared. Attributes pertaining to Ligule, Collet and Scutellum have been found significant for differentiation of reported grass species at seedling stage.
\end{abstract}

Copy Right, IJAR, 2016,. All rights reserved.

\section{Introduction:-}

The genus Digitaria Heist. Ez. Fab. (Panicoideae, Paniceae, Poaceae), commonly known as digit grass. The name Digitaria is derived from the Latin digitus (finger), alluding to radiating inflorescence branches (Watson and Dallwitz, 1992; Quattrocchi, 2006). Hence, Digitaria species are collectively called "finger grasses". Heister (1748) gave the name Digitaria for the first time and separated it from the genus Panicum. Haller, in 1768, validated the genus and gave a detailed description (Henrard, 1950).

Digitaria with around 218 species (Vega et al., 2009) is the third most speciose genera within tribe Paniceae (Clayton and Renvoize, 1986). There is considerable diversity both between and within Digitaria species, which may be annual or perennial, with or without rhizomes, with or without stolons, and tufted (erect) or decumbent or sometimes prostrate (sward/mat forming). The genus is extremely variable with regard to inflorescence structure, relative length of spikelet scales, and spikelet indumentum types, with different combinations of appressed pubescence, glassy bristles and marginal cilia possible within the same species or even between the two members of a spikelet pair. Despite this, the overall facies of the spikelet is surprisingly uniform, and the genus is seldom difficult to recognise (Clayton and Renvoize, 1986). Based on a recent study of phylogeny in the tribe Paniceae by Morrone et al. (2012), the genus Digitaria appears to be monophyletic. Basic chromosome numbers in the genus Digitaria are $\mathrm{x}=9,15$ and 17, the most frequent chromosome numbers being $2 \mathrm{n}=18,36,54$ and 72 , but variants with $24,27,30,34,35,40,45,60,68,70$ and 76 have also been reported, as well as intra-specific variability in chromosome number (Pozzobon et al., 2006).

The species of Digitaria are meso- or xerophytic, generally growing in open habitats and in a wide range of environments, including sandy beaches. Some species are very weedy, e.g. the well known crab grasses $D$. ciliaris and D. sanguinalis, but other taxa may also be invasive, such as $D$. abyssinica, $D$. adscendens, $D$. fuscescens, $D$. horizontalis, D. ischaemum, D. longiflora, D. radicosa, D. scalarum, D. setigera, D. ternata and D. violascens. Holm et al. (1977) lists the annual D. sanguinalis (L.) Scop. (crabgrass) and the perennial D. abyssinica (Hochst. ex 
A. Rich.) Stapf among the world's 100 worst weeds. Some are cultivated as forages, e.g. Pangola grass, classically known as D. decumbens, recently renamed $D$. eriantha ssp. pentzii (Zuloaga et al. 1994), D. milanjiana, D. smutsii and $D$. eriantha. Others, such as $D$. aridicola, $D$. didactyla, D. gazensis, D. longiflora, D. milanjiana, D. nodosa, $D$. pearsonii, D. velutina are components of native pastures, or are cultivated for lawn, e.g. D. didactyla, D. longiflora, D. swazilandensis and D. timorensis (Watson and Dallwitz, 1992). Some of the invasive species such as $D$. sanguinalis may be used as grain crops in times of shortage and there are records of the utilisation of this species in historical times. Other taxa such as D. cruciata, D. ciliaris, D. longiflora and D. exilis (Fonio) are still explored as minor crops in some localities of Asia and Africa (De Wet, 1995). Fonio is sometimes regarded as "grain of life" as it provides food early in the farming season, when other crops are yet to mature for harvest (Ibrahim, 2001).

With the establishment of Grass Phylogeny Working Group (GPWG, 2001), we witnessed a great upsurge in research on various aspects of grasses across the globe. Amidst a scenario of renewed interest in various aspects of grasses, India has been identified, as a 'seriously under-collected country' as far as inventorization of grass diversity is concerned (Kellogg, 2006).

The present study area i.e. the Vindhyan region consists of the Vindhyan plateau and hills in the southern part of the Uttar Pradesh state. The topography consists mainly of plateau lands but has lot of local variations covering Chandauli, Mirzapur and Sonbhadra districts $\left(24^{\circ} 50^{\prime}\right.$ and $25^{\circ} 35^{\prime} \mathrm{N}$ latitude and $82^{\circ} 50^{\prime}$ and $83^{\circ} 35^{\prime}$ E longitude, sea level height 90 to $300 \mathrm{mts}$.).

The climate is dry sub-humid with three distinct seasons viz. winter, summer and rainy and is characterized by long and intensely hot summer, low rainfall and a short mild winter. The hot weather usually begins from middle of March and extends to the break of the monsoon in the month of June-July. The summer temperature goes up to $48^{\circ} \mathrm{C}$ in May-June. However, the average temperature varies from $8^{\circ} \mathrm{C}$ to $34^{\circ} \mathrm{C}$. The rainfall varies from $750 \mathrm{~mm}$. to about $1200 \mathrm{~mm}$. Most of the rainfall occurs in the months of June, July and August. There is little winter rains, which occurs generally in January and February.

The soil of the study area varies considerably but most of the area has red lateritic soils with often-pronounced nodules locally called "Murram". Mirzapur and some part of other districts of this region have red soil. Deep and fertile soils are often found in the valleys between folds of hills where the soil washed from hill slopes has accumulated. Hence, the topography presents a contrast from bare rocky lands alternating with good deep soils.

In the present study an attempt has been made to record the taxonomy of six congeneric taxa of grasses belonging to the genus Digitaria alongwith their seedling morphology, occurring in Vindhyan region of Uttar Pradesh, India. Key to the mature plants as well as seedlings and illustrations have been also been provided.

\section{Materials and method:-}

While working on Grass flora of Vindhyan Region, Uttar Pradesh, India, six taxa of the genus Digitaria have been collected at different growth stages along with their seeds. To workout the taxonomy of collected specimens authentic Floras and related publications have been consulted. The seeds of collected specimens have been grown in petri dishes $(20 \times 5 \mathrm{~cm})$ on moist filter paper at $22-24^{\circ} \mathrm{C}$ in permanent light and in sandy soil in the green house. Morphological features of seedlings have been described as per Singh et al., 2013, using Stereoscopic Binocular Microscope (Olympus-Magnus MSZ-Bi) . Voucher specimens have been deposited in the Herbarium, Department of Botany, Udai Pratap College (Autonomous), Varanasi, India.

\section{Results:-}

\section{Key to the mature specimens:-}

1. Nodes pubescent, Upper glume 7 nerved. Caryopsis light yellow.................................... ciliaris

1.Nodes glabrous, Upper glume 3-5 nerved. Caryopsis light brown/light purple/light green..........................2

2. Leaf blade glabrous, Ligule apex obtuse.

D. ternata

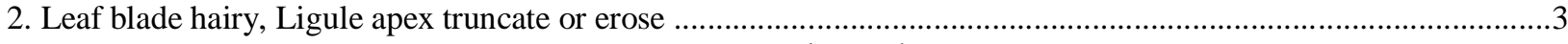

3.Leaf sheath compact, Prophyllum deltoid, Upper glume trinerved................................. abludens

3.Leaf sheath loose, Prophyllum lanceolate, Upper glume pentanerved..........................................

4.Leaf blade scabrous, Racemes 6-8 in number, Caryopsis oblong .............................. violascens

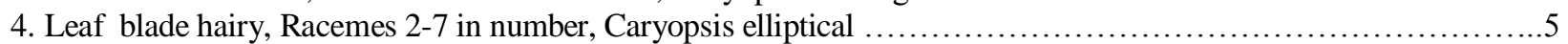

5.Rachis flat but Pedicel terete. Spikelets hairy, Lower glume absent........................................................ longiflora 


\section{Key to the Seedling:-}

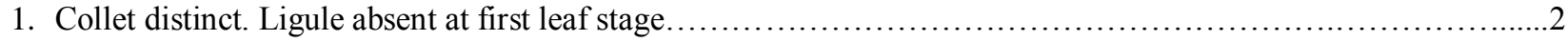

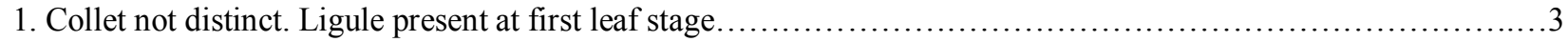

2. Scutellum hemispherical, Coleoptile loose........................................................................................ abludens

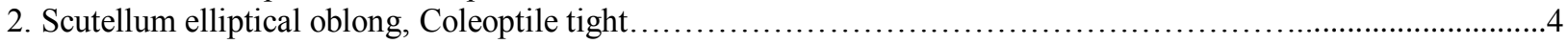

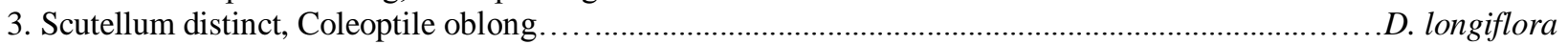

3. Scutellum not distinct, Coleoptile obovate.......................................................................................... griffithii

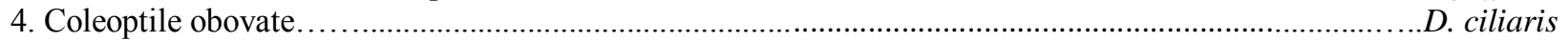

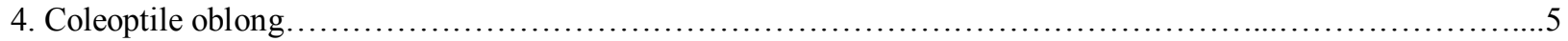

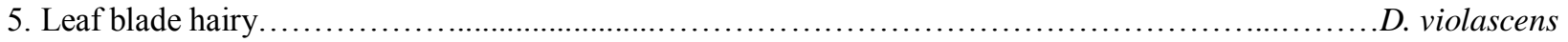

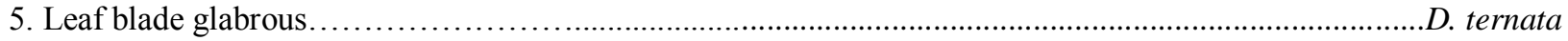

\section{Systematic Enumeration:-}

D. abludens (Roem. \& Schult.) Veldkamp in Blumea 21:53. 1973; Schult. Syst. Veg. 2:457. 1817. Digitaria granularis (Spreng.) Henr. Monogr. Dig. 302.1950; Bor, Grass Bur. Cey. Ind. Pak. 31.1960; Raizada \& Jain, Indian Forest. Rec. Ser. 2 Bot. 5:187.1964; Rajagopal \& Panigrahi, Proc. Nat. Acad. Sci. Ind. Sec. B 37:33.1967; Uniyal et.al. The Grasses of U.P.-A checklist.40.1994; Moulik, Grass. Bamb. Ind. 1:90.1997. D. pedicelllaris (Trin. ex Hook.f.) Prain, Bengal Pl.1191.1903; Haines, Bot. Bihar \& Orissa. 1009. 1924; Fischer in Gamble, Fl. Pres. Madr. 3:1223.1957. (Fig. 1A).

An annual grass. Root fibrous, dull white, tufted, $8.0-10.0 \mathrm{~cm}$ long. Culms green, glabrous, ascending, terete, 30.0$40.0 \mathrm{~cm}$ long. Nodes green, glabrous, slightly swollen. Internodes $5.0-7.0 \mathrm{~cm}$ long. Leaf sheath light green, membranous, tight, hairy; hair base broad, apex acute, $0.6 \mathrm{~cm}$ long; margins entire, hyaline, glabrous. Collar light green, hairy; hair base broad, apex acute, $0.6 \mathrm{~cm}$ long. Auricle absent. Ligule oblong, membranous, base and apex truncate, glabrous, $0.1 \mathrm{~cm}$ long. Leaf blade lanceolate, $8.0 \times 0.5 \mathrm{~cm}$, mean length/width $(1 / \mathrm{w}) 16$, base truncate, apex acuminate, margins membranous, denticulate, hairy only at base; hairs as on collar, surfaces green, membranous, scabrous and glabrous. Venation parallelodromous; multicostate reticulate, veins-7. Prophyllum deltoid, 1.0 x 0.40 $\mathrm{cm}$, mean 1/w 2.5, base truncate, apex acute, margins hyaline, transparent, glabrous, light green, membranous, binerved; nerves green, distinct and winged; wings hyaline, scabrous, hairy; hairs as on leaf sheath. Inflorescence raceme; racemes linear, digitate, scattered, green, 4-5 in number, 5-6 cm long. Rachis green, triquetrous, scabrid. Spikelets in pair, one sessile and other pedicelled; pedicel green, triquetrous, scabrid. Spikelets elliptical, $0.25 \times 0.1$ $\mathrm{cm}$, mean 1/w 2.5, base rounded, apex acute, green, hairy; base broad, apex acute. Lower glume abscent. Upper glume ovate, $0.15 \times 0.10 \mathrm{~cm}$, mean 1/w 1.5, base truncate, apex obtuse, membranous, green, hairy; hairs as on spikelet; trinerved; nerves green, distinct, glabrous. Lower lemma oblong, $0.3 \times 0.15 \mathrm{~cm}$, mean 1/w 2, base truncate, apex acute, green, membranous, glabrous, trinerved; nerves green, distinct, glabrous. Upper lemma oblong, $0.23 \mathrm{x}$ $0.15 \mathrm{~cm}$, mean 1/w 1.2, base truncate, apex obtuse, margins membranous, glabrous, light green, chartaceous, nerveless. Upper palea oblong, $0.20 \times 0.10 \mathrm{~cm}$, mean 1/w 2, base truncate, apex obtuse, margins membranous, entire, light green, nerveless. Lodicules absent. Stamens 3; anthers dithecous, versatile, dorsifixed, elliptical, green, glabrous, $0.2 \mathrm{~cm}$ long. Ovary ovate, glabrous, translucent, $0.1 \mathrm{~cm}$ long; styles 2, linear, terete, glabrous, $0.1 \mathrm{~cm}$ long ; stigma light pink, plumose. Caryopsis obovate, $0.15 \times 0.10 \mathrm{~cm}$, mean 1/w 1.5, base and apex obtuse, light brown and glabrous.

Seedling mesogeal. Primary root fibrous, less developed than shoot, less branched, dull white and hairy, terete, 0.3 $\mathrm{cm}$ long at coleoptile stage, and $0.6 \mathrm{~cm}, 0.9 \mathrm{~cm}$ and $1.8 \mathrm{~cm}$ long at $1^{\text {st }}, 2^{\text {nd }}$ and at $3^{\text {rd }}$ leaf stages respectively. Collet dull white, hairy and swollen. Scutellum hemispherical, grey and glabrous. Mesocotyl white, glabrous, terete, $1.0 \mathrm{~cm}$ long. Mesocotyl roots not distinct. Coleoptile oblong, base truncate, apex obtuse, translucent, loose, binerved, 0.4 $\mathrm{cm}$ long. Internodes (at $3^{\text {rd }}$ leaf stage): First one $1.0 \mathrm{~cm}$ long, later internodes elongating. Leaf sheath green, sparsely hairy; hair base broad, apex acute, transparent, unicellular, $0.1 \mathrm{~cm}$ long; margins scabrous, $0.4 \mathrm{~cm}$ long. Ligule absent. First leaf blade oblanceolate-linear, $3.5 \times 0.5 \mathrm{~cm}$, mean L/W 7.0, base attenuate, apex acute, margins scabrous, both surfaces green and sparsely hairy; hairs as on leaf sheath. Venation parallelodromous; multicostate striated, several primary veins distinct, reaches to blade apex. Second and subsequent leaves same as that of $1^{\text {st }}$ leaf but leaf blade linear. 
Flowering \& Fruiting:- September-October.

Distribution: India, Sri Lanka, Tropical Australia.

D. ciliaris (Retz.) Koel. Descr. Gram. 27:1802, Clayton in Fl. Trop. Afr. 2,3:453.1972; Veldkamp in blumea 21:32.1972; Cope in Nasir \& Ali, Fl. W. Pak.143:228.1982; Manilal \& Sivaraj, Fl. Calic. 335.1982; Uniyal et.al. The Grasses of U.P.-A checklist.40.1994; Moulik, Grass. Bamb. Ind. 1:90.1997. D. adscendens (Kunth) Henr. Blumea 1:92.1834, excl. ssp. chrysoblephara Hack. Monogr.Digi 9:1950; Bor, Grass Bur. Cey. Ind. Pak. 298.1960; Raizada \& Panigrahi, Proc. Nat. Acad. Sci. Ind. Sec. B 37:32.1967. (Fig. 1B).

An annual grass. Root fibrous, dull white, $8.0-10.0 \mathrm{~cm}$ long. Culms green, tufted, erect, laterally compressed, ascending, 40-50 cm long. Nodes light green, pubescent; hair base broad, apex acute, transparent, $0.1 \mathrm{~cm}$ long. Internodes 5.0-6.0 cm long. Leaf sheath green, membranous, scabrous and pubescent; hairs as on nodes; margins glabrous, 4.0-5.0 cm long. Collar green, pubescent; hairs as on leaf sheath. Auricle absent. Ligule membranous, base truncate, apex rounded, glabrous, $0.15 \mathrm{~cm}$ long. Leaf blade liniar-lanceolate, $7.0 \times 0.5 \mathrm{~cm}$, mean 1/w 14, base truncate, apex acuminate, margins denticulate, green, membranous, scabrous, hairy only at base; hairs base broad, apex acute, $0.2 \mathrm{~cm}$ long. Venation parallelodromous; multicostate reticulate, 7 veins distinct. Prophyllum lanceolate, $1.0 \times 0.20 \mathrm{~cm}$, mean 1/w 5.0, base truncate, apex obtuse, margins hyaline, transparent, light green, membranous, glabrous, binerved; nerves green, distinct, winged and scabrous. Inflorescence racemes: racemes linear, digitate, scattered, green, 2-3 in number, 5-6 cm long. Rachis green, triquetrous, scabrid. Spikelets in pair, one sessile, other pedicelled; pedicel green, triquetrous; scabrid, $0.2 \mathrm{~cm}$ long. Spikelets ovate, $0.25 \times 0.12 \mathrm{~cm}$, mean 1/w 2.1, base rounded, apex obtuse, green, hairy; hairs as on rachis. Lower glume ovate, $0.20 \times 0.10 \mathrm{~cm}$, mean 1/w 2.0, base truncate, apex rounded, margins membranous, hairy; hairs as on spikelets; green, membranous, glabrous, trinerved; nerves green, glabrous. Upper glume elliptical, $0.25 \times 0.12 \mathrm{~cm}$, mean 1/w 2.1, base truncate, apex acute, margins as on lower glume, green, membranous, hairs in two rows; hairs as on lower glume; 7-nerved; nerves green, distinct. Lemma elliptical, $0.20 \times 0.10 \mathrm{~cm}$, mean $1 / \mathrm{w} 2$, base truncate, apex obtuse, margins entire, light green, membranous, glabrous, trinerved; nerves light green, not distinct. Palea elliptical, $0.18 \times 0.10 \mathrm{~cm}$, mean 1/w 1.8, base truncate, apex obtuse, hyaline, light green, glabrous, nerveless. Lodicules absent. Stamens 3, anthers dithecous, versatile, dorsifixed, lanceolate, glabrous, green, $0.15 \mathrm{~cm}$ long. Ovary ovate, glabrous, translucent and $0.05 \mathrm{~cm}$ long; styles 2 , linear, terete, glabrous, $0.10 \mathrm{~cm}$ long; stigma transparent, hairy; hairs in two rows. Caryopsis elliptical, $0.20 \mathrm{x} 0.10$ $\mathrm{cm}$, mean 1/w 2, base and apex obtuse, white-yellow, glabrous.

Seedling mesogeal. Primary root fibrous, less developed than shoot, less branched, dull white and hairy, terete, 1.0 $\mathrm{cm}$ long at coleoptile stage, and $1.3 \mathrm{~cm}, 1.6 \mathrm{~cm}$ and $2.5 \mathrm{~cm}$ long at $1^{\text {st }}, 2^{\text {nd }}$ and at $3^{\text {rd }}$ leaf stages respectively. Collet distinct, white, glabrous and slightly swollen. Lemma elliptical, $0.3 \times 0.1 \mathrm{~cm}$, mean L/W 3.0, base and apex acute, villose; hairs unicellular, cylindrical, $0.1 \mathrm{~cm}$ long; trinerved and grey. Palea elliptic, $0.25 \mathrm{x} 0.15 \mathrm{~cm}$, mean L/W 1.6, base and apex acute, margins entire and infolded, glabrous and binerved. Scutellum not distinct. Mesocotyl distinct, dull white, glabrous, terete in cross section, fistular, $0.7 \mathrm{~cm}$ long. Mesocotyl roots not distinct. Coleoptile obovate, transparent, base attenuate, apex acute, compressed, glabrous, tight and binerved, $0.7 \mathrm{~cm}$ long. Internodes (at $3^{\text {rd }}$ leaf stage): First one $0.3 \mathrm{~cm}$ long, later internodes elongating. Leaf sheath green, hairy; hairs as on lemma; margins scabrous, $0.4 \mathrm{~cm}$ long. Ligule appear after $1^{\text {st }}$ leaf stage, membranous, apex obtuse, $0.2 \mathrm{~cm}$ long. Leaf blade elliptical, $1.0 \times 0.4 \mathrm{~cm}$, mean L/W 2.5, base attenuate, apex acute, margins scabrous, both surfaces green and hairy; hairs as on lemma. Venation parallelodromous; multicostate striated, several primary veins distinct, each vein reaches to blade apex. Second and subsequent leaves same as that of $1^{\text {st }}$ leaf but leaf blade linear.

Flowering \& Fruiting:- August-November.

Distribution: Burma, India, Malaya.

D. griffithii (Hook.f.) Henr.in Blumea.1:100.1934; Fischer in Gamble, Fl. Pres. Madr. 3:1122.1957; Bor, Grass Bur. Cey. Ind. Pak.301.1960; Uniyal et.al. The Grasses of U.P.-A checklist.42.1994; Moulik, Grass. Bamb. Ind. 1:93.1997.-D.sanguinalis (L.) Scop. Var. griffthii (Hook.f.) Rang. \& Tad. S. Ind. Grass. 54.t.73.1921.-Paspalum sangunalis (L.) Lamk. Tab. Encycl. Meth. Bot. 1: 176.1791; Hook.f. Fl. Brit. Ind. 7:15.1896. Panicum corymbosum Thw. Enum. Pl. Zeyl.436.1864. P. sanguinale L. Sp. Ed. 1:57.1753. (Fig. 1C)

An annual or perennial grass. Roots fibrous, dull white with secondary roots at lower nodes, $12-15 \mathrm{~cm}$ long. Culms green, glabrous, tufted, ascending, $70-100 \mathrm{~cm}$ long. Nodes brown, glabrous and slightly swollen. Internodes $6.0 \mathrm{~cm}$ 
long. Leaf sheath green, membranous, pubescent; hair base broad, apex acute, transparent, $0.1 \mathrm{~cm}$ long; margins glabrous, 4.0-5.0 cm long. Collar light green, hairy; base broad, apex acute and $0.6 \mathrm{~cm}$ long. Auricle absent. Ligule membranous, glabrous, base truncate, apex erose, margins entire, $0.2 \mathrm{~cm}$ long. Leaf blade lanceolate, $8.0 \times 0.5 \mathrm{~cm}$, mean 1/w 16, base truncate, apex acuminate, margins membranous, denticulate, hairy; hairs as on leaf sheath; green, membranous, scabrous, hairy; hairs as on leaf sheath. Venation parallelodromous; multicostate reticulate, veins - 7 . Prophyllum lanceolate, $1.0 \times 0.20 \mathrm{~cm}$, mean 1/w 5, base truncate, apex obtuse, glabrous, light green, transparent, binerved; nerves green, distinct, scabrid. Inflorescence racemes; racemes linear, digitate, compact, green, 5-7 in number, 8-10 cm long. Rachis green, triquetrous, minutely winged, scabrid. Spikelets in pair, one sessile, other pedicelled; pedicel green, triquetrous, scabrid, $0.2 \mathrm{~cm}$ long. Spikelets elliptical, $0.40 \times 0.15 \mathrm{~cm}$, mean 1/w 3.3, base rounded, apex acute, green, glabrous. Lower glume ovate, $0.25 \times 0.12 \mathrm{~cm}$, mean 1/w 2.1, base truncate, apex obtuse, margins hyaline, hairy; hairs as on leaf blade; light green, membranous, trinerved; nerves green, distinct. Upper glume obovate, $0.35 \times 0.20 \mathrm{~cm}$, mean $1 / \mathrm{w} 1.7$, base truncate, apex obtuse, green, membranous, glabrous, pentanerved; nerves green, distinct, glabrous. Lower lemma elliptical, $0.25 \times 0.15 \mathrm{~cm}$, mean 1/w 1.6, base rounded, apex obtuse, green, chartaceous, pentanerved; nerves cnot distinct. Upper lemma elliptical, $0.25 \times 0.15 \mathrm{~cm}, \mathrm{mean} 1 / \mathrm{w}$ 1.6, base truncate, apex obtuse, green, membranous, glabrous, pentanerved; nerves green, distinct, glabrous. Upper palea ovate, $0.20 \times 0.1 \mathrm{~cm}$, mean 1/w 2, base truncate, apex acute, margins inrolled, hyaline, glabrous, binerved; nerves light green, glabrous. Lodicules absent. Stamenes3 ; anthers dithecous, versatile, dorsifixed, ovate, glabrous, light green, base rounded, apex acute, $0.30 \mathrm{~cm}$ long. Ovary ovate, glabrous, translucent, $0.1 \mathrm{~cm}$ long; styles 2 , linear, terete, glabrous, $0.05 \mathrm{~cm}$ long; stigma transparent and hairy, hairs in two row. Caryopsis elliptical, $0.15 \mathrm{x} 0.10 \mathrm{~cm}$, mean $1 / \mathrm{w} 1.5$, base rounded, apex obtuse, white-brown and glabrous.

Seedling mesogeal. Primary root fibrous, less developed than shoot, less branched, white and glabrous, terete in cross section, $0.1 \mathrm{~cm}$ long at coleoptile stage, and $0.7 \mathrm{~cm}, 1.1 \mathrm{~cm}$ and $1.9 \mathrm{~cm}$ long at $1^{\text {st }}, 2^{\text {nd }}$ and at $3^{\text {rd }}$ leaf stages respectively. Collet and Scutellum not distinct. Mesocotyl distinct, translucent, white, glabrous, terete in cross section, fistular, $1.2 \mathrm{~cm}$ long. Coleoptile obovate, transparent, glabrous, base attenuate, apex obtuse, binerved, 0.8 $\mathrm{cm}$ long. Leaf sheath green, hairy; hairs multicellular, cylindrical, brown, $<0.1 \mathrm{~cm}$ long; margins scabrous, $0.3 \mathrm{~cm}$ long. Ligule membranous, apex erose, $<0.1 \mathrm{~cm}$ long. Leaf blade oval-elliptical, 0.7 x $0.4 \mathrm{~cm}$, mean L/W 1.7, base attenuate, apex rounded, margins scabrous, both surfaces green and hairy; hairs as on leaf sheath. Venation parallelodromous; multicostate striated, several primary veins distinct, reaches to blade apex. Second and subsequent leaves same as that of $1^{\text {st }}$ leaf but leaf blade linear.

Flowering \& Fruiting:- May-September.

Distribution: Burma, Malaysia, Southern India, Sri Lanka.

D. longiflora (Retz.) Pers.Syn.Pl.1:85.1805; Hern. Mongr. Dig.405.1905; Haines, Bot. Bihar \& Orissa. 1008. 1924; Fischer in Gamble, Fl. Pres. Madr. 3:1223.1957; Bor, Grass Bur. Cey. Ind. Pak.302.1960; Raizada \& Jain, Indian Foret. Rec. Ser. 2. Bot.5: 194.1964; Rajagopal \& Panigrahi, Proc. Nat. Acad. Sci. Ind. Sec. B 37:33.1964,Veldkamp in blumea 21:66.1972; Cope in Nasir \& Ali, Fl. W. Pak.1434:219.1982; Manilal \& Sivaraj, Fl. Calic. 335.1982; Uniyal et.al. The Grasses of U.P.-A checklist.42.1994; Moulik, Grass. Bamb. Ind. 1:93.1997. (Fig 1D).

An annual grass. Root fibrous, dull white, $8-12 \mathrm{~cm}$ long. Culms green, glabrous, ascending, slightly compressed, 50-60 cm long. Nodes green, glabrous. Internodes $6.0 \mathrm{~cm}$ long. Leaf sheath green, membranous, pubescent; hair base broad, apex acute, transparent, unicellular, $0.2 \mathrm{~cm}$ long; margins glabrous and 4.0-5.0 cm long. Collar light green, hairy; hairs as on leaf sheath. Auricle absent. Ligule membranous, glabrous, base truncate, apex erose, glabrous, $0.1 \mathrm{~cm}$ long. Leaf blade lanceolate, $8.0 \times 0.70 \mathrm{~cm}$, mean 1/w 11.4, base truncate, apex acuminate, margins membranous, denticulate, hairy; hair base tubercled, apex acute, transparent, $0.2 \mathrm{~cm}$ long; green. Venation parallelodromous; multicostate reticulate, veins-11. Prophyllum lanceolate, $1.0 \times 0.20 \mathrm{~cm}$, mean 1/w 5, base truncate, apex acute, margins hyaline, transparent, glabrous; green, membranous, glabrous, and binerved ; nerves green, distinct, scabrid and winged. Inflorescence racemes: racemes linear, digitate, scattered, green, 2-3 in number, 8-10 $\mathrm{cm}$ long. Rachis green, flate, broadly winged, scabrid. Spikelets in pair, one sessile and other pedicelled, Pedicel green, terete, glabrous. Spikelets elliptical, $0.30 \times 0.15 \mathrm{~cm}$, mean 1/w 2, base rounded, apex acute, green, softely hairy; hair base broad, apex acute, transparent, unicellular, $0.2 \mathrm{~cm}$ long. Lower glume absent. Upper glume ovate, $0.20 \times 0.10 \mathrm{~cm}$, mean 1/w 2, base truncate, apex acute, membranous, hairy; hair base broad, apex acute, transparent, unicellular, $0.1 \mathrm{~cm}$ long; green, pentanerved; nerves green, visible, glabrous, ending at apex. Lower lemma elliptical, $0.30 \times 0.10 \mathrm{~cm}$, mean 1/w 3, base rounded, apex acute, green, membranous, hairy; hairs as on upper glume, trinerved; nerves green, distinct, glabrous. Upper lemma ovate, $0.20 \times 0.10 \mathrm{~cm}$, mean 1/w 2, base rounded, apex acute, glabrous, green, membranous, coriaceous, trinerved; nerves green, distinct. Upper palea elliptical 0.20 x 0.10 
$\mathrm{cm}$, mean 1/w 2, base truncate, apex denticulate coriaceous, translucent, binerved; nerves green. Lodicules absent. Stamenes 3; anthers dithecous, versatile, dorsifixed, oblong, glabrous, light yellow, base rounded, apex acute, 0.20 cm long. Ovary ovate, glabrous, translucent, $0.1 \mathrm{~cm}$ long; styles 2, linear, terete, glabrous, $0.05 \mathrm{~cm}$ long; stigma transparent, hairy; hair base broad, apex acute, in two row. Caryopsis elliptical, 0.10 x $0.05 \mathrm{~cm}$, mean 1/w 2, planoconvex, base rounded, apex obtuse, surfaces pale brown, glabrous.

Seedling mesogeal. Primary root fibrous, less developed than shoot, less branched, dull white and hairy, terete, 0.5 $\mathrm{cm}$ long at coleoptile stage, and $0.8 \mathrm{~cm}, 1.9 \mathrm{~cm}$ and $2.3 \mathrm{~cm}$ long at $1^{\text {st }}, 2^{\text {nd }}$ and at $3^{\text {rd }}$ leaf stages respectively. Collet not distinct. Scutellum conical, grey-white, and glabrous. Mesocotyl distinct, white, glabrous, terete, $0.3 \mathrm{~cm}$ long. Mesocotyl roots not distinct. Coleoptile oblong, transparent, base truncate, apex obtuse, glabrous, and binerved, $0.5 \mathrm{~cm}$ long. Leaf sheath green, hairy; hairs as on lemma; margins keeled, $0.2 \mathrm{~cm}$ long. Ligule membranous, apex erose, $0.05 \mathrm{~cm}$ long. Leaf blade elliptical, $1.0 \times 0.3 \mathrm{~cm}$, mean L/W 3.3, base attenuate, apex acute, margins keeled, both surfaces green and hairy; hairs unicellular, cylindrical, transparent, $<0.1 \mathrm{~cm}$ long. Venation parallelodromous; multicostate striated, several primary veins distinct, reaches to blade apex. Second and subsequent leaves same as that of $1^{\text {st }}$ leaf but leaf blade linear.

Flowering \& Fruiting:- October-January.

Distribution: India, Malaysia, Madagascar, Mascarnes, Tropical \& South Africa.

D. ternata (A. Rich.) Stapf. in Dyer. F1. Cap.7: 376. 1898; Stapf in F1. Trop. Afr. 9: 452. 1919 ; Cook, F1. Bomb.2:940. 1908; Bor, Gass. Bur.Cey. Ind. Pak. 306.1960; Blatter \& McCann, Bomb. Grass. 124.f.77.1984; Moulik, Grass. Bomb. Ind. 1: 97 .1997. Cynodon ternatum A. Rich. Tent. F1. Abyss.2: 405.1851. Paspalum ternatum Hook. f.F1. Brit. Ind.7:17.1896; Lisboa, Bomb. Grass. 4.1896. Panicum ternatum Hochst. in Flora 25: 19.1841; Hack. in Oest. Bot. 1:40.1854; Hochst. ex Steud., Syn. Pl. Glum. 1: 40. 1854. P. Phanerocarpum var. gracile Nees. F1. Aftr. Austr. 23.1841. Syntherisma ternata (Itochst.) Newbold I Torreya 24:9.1924. (Fig 1E)

Annual grass. Roots fibrous, dull white, $10-12 \mathrm{~cm}$ long. Culms green, glabrous, tufted, ascending, $50-60 \mathrm{~cm}$ long. Nodes green, glabrous and slightly swollen. Internodes $8.0 \mathrm{~cm}$ long. Leaf sheath green, membranous, compact, glabrous, 4.0-5.0 cm long. Collar light green, hairy; hair base broad, apex acute and $0.6 \mathrm{~cm}$ long. Auricle absent. Ligule membranous, glabrous, base truncate, apex obtuse, margins entire, $0.2 \mathrm{~cm}$ long. Leaf blade lanceolate, $8.0 \mathrm{x}$ $0.5 \mathrm{~cm}$, mean 1/w 16, base truncate, apex acuminate, margins denticulate, only base hairy; hairs as on collar; green, membranous, scabrous. Vanation parallelodromous; multicostate, reticulate, veins-9. Prophyllum lanceolate, $1.0 \mathrm{x}$ $0.20 \mathrm{~cm}$, mean 1/w 5, base truncate, apex obtuse, margins hyaline, green, membranous, glabrous, binerved; nerves green, distinct and winged. Inflorescence racemes: racemes linear, digitate, green, lax, 4-6 in number, 5-6 cm long. Rachis green, triquetrous, scabrid, broadly winged. Spikelets in 3-4 groups, one sessile, other pedicelled; pedicel green, triquetrous, scabrid, $0.2 \mathrm{~cm}$ long. Spikelets ovate, $0.25 \times 0.12 \mathrm{~cm}$, mean 1/w 2.1, base rounded, apex obtuse, green, hairy; hairs clavellate type. Lower glume absent. Upper glume elliptical, 0.10 x $0.05 \mathrm{~cm}$, mean 1/w 2.0, base truncate apex rounded, margins hyaline, glabrous, green, membranous, hairy; hairs as on spikelets; single nerved. Lower lemma ovate, 0.15 x $0.10 \mathrm{~cm}$, mean 1/w 1.5, base truncate, apex obtuse, margins light green, glabrous, green, membranous, hairy; hairs as on spikelet; pentanerved; nerves green, distinct, hairy; hairs as on lower glume. Upper Lemma elliptical, $0.15 \times 0.10 \mathrm{~cm}$, mean $1 / \mathrm{w} 1.5$, base truncate, apex acute, margins entire, green, membranous, glabrous, trinerved; nerves green, glabrous, not distinct. Upper palea elliptical, $0.10 \times 0.08 \mathrm{~cm}$, mean 1/w 1.2 , base truncate, apex obtuse, hyaline, translucent, glabrous, binerved; nerves, light green, glabrous, not distinct. Lodicules2 , obovate, base obtuse, apex truncate and unduelate, translucent, glabrous, $0.15 \mathrm{~cm}$ long. Stamenes-3; anthers dithecous, versatile, dorsifixed, ovate, glabrous, light green, base rounded, apex acute, $0.30 \mathrm{~cm}$ long. Ovary obovate, glabrous, translucent, $0.1 \mathrm{~cm}$ long; styles 2, linear, terete, glabrous, $0.05 \mathrm{~cm}$ long; stigma transparent, fimbriate. Caryopsis obovate, $0.10 \times 0.06 \mathrm{~cm}$, mean 1/w 1.6, base and apex obtuse, light brown, glabrous.

Seedling mesogeal. Primary root fibrous, branched, dull white, terete and $1.50 \mathrm{~cm}$ long at coleoptile stage, $2.0 \mathrm{~cm}$, $2.5, \mathrm{~cm}$ and $3.0 \mathrm{~cm}$ long at $1^{\text {st }}, 2^{\text {nd }}$, and $3^{\text {rd }}$ leaf stages respectively. Collet distinct, brown and hairy; hairs base broad, apex acute and $0.1 \mathrm{~cm}$ long. Scutellum elliptical, 0.20 x $0.05 \mathrm{~cm}$, mean 1/w 4.0, base acute, apex rounded, dull white, surface flate and wavy. Mesocotyl light green, glabrous, terete and $0.2 \mathrm{~cm}$ long. Coleoptile oblong, $0.60 \mathrm{x}$ $0.25 \mathrm{~cm}$, mean 1/w 2.4, base truncate, apex obtuse, green, hyaline, glabrous and binerved. Adventitious roots appear after first leaf stage. First leaf sheath green, membranous, oblong, margins hyaline and glabrous, green, membranous and hairy; hair base broad and apex acute. Ligule appears after $1^{\text {st }}$ leaf stage. First leaf blade elliptical, $0.60 \mathrm{x} 0.20$ $\mathrm{cm}$, mean 1/w 3.0, base acute, apex obtuse, margins denticulate, green, membranous, glabrous, veins - 5. Second leaf 
sheath same as that of first leaf sheath. Ligule membranous, glabrous and entire. Second leaf blade lanceolate, $1.0 \mathrm{x}$ $0.22 \mathrm{~cm}$, mean 1/w 5, base truncate, apex acute, margins denticulate, green, membranous, scabrid and veins 5 .

Flowering \& Fruiting:- September-December

Distribution: India, Tropical and South Africa, China

D. violascens (Link.) Hort. Berol. 1: 229. 1827; Henr. Monogr.Digi. 790. 1950; Moulik, Grass. Bomb. ind. 1:97.1997; Bor, Grass. Bur. Cey. Ind. Pak. 1960; Rajagopal \& panigrahi, Proc. Nat. Acad. Sci. India Sec. B. 37: 35. 1967; Gilliland in F1. Malaya. 3; 191. 1971; Veldkamp in Blumia 21: 63. 1973; Cop in Nasir \& Ali, F1. W. Pak. 143: 219. 1960. D. chinensis ( Nees) . Camus in Not. Syst. 4: 48. 1923, non Hornem 1819; Fischer in Gamble, F1. Pres. Madr. 1765. 1934 (3: 1223. 1957 repr. ed.). Paspalum chinensis Nees in Hook. \& Arn. Bot. Beech. Voy. 231. 1841. P. longifloram acut. non Retz. 1786; Hook. f. F1. Brit. Ind. 7: 17. 1896. (Fig. 1F).

An annual grass. Roots fibrous, branched, dull white, $8.0-10.0 \mathrm{~cm}$ long. Culms green, glabrous, tufted, erect, decumbent, 40.0-50.0 cm long. Nodes green, glabrous. Internodes $8.0 \mathrm{~cm}$ long. Leaf sheath green, membranous, loose, glabrous, $6.0-7.0 \mathrm{~cm}$ long. Collar light green, glabrous. Auricle absent. Ligule membranous, glabrous, base and apex truncate, $0.20 \mathrm{~cm}$ long. Leaf blade lanceolate, $12.0 \times 0.80 \mathrm{~cm}$, mean 1/w 15, base truncate, apex acuminate, margins denticulate,green, membranous, scabrous. Vanation parallelodromous; multicostate reticulate, veins-11. Prophyllum lanceolate, $1.5 \times 0.25 \mathrm{~cm}$, mean $1 / \mathrm{w} 6$, base truncate, apex acute, margins hyaline, scabrous, green, membranous, glabrous, binerved; nerves green, distinct and winged. Inflorescence racemes: racemes green, linear, digitate, compact, 6-8 in number, 10.0-12.0 cm long. Rachis green, flate, scabrid, mid vain prominent, winged. Spikelets in pair, one sessile and other pedicelled; pedicel green, terete; scabrid, $0.2 \mathrm{~cm}$ long. Spikelets elliptical, $0.30 \times 0.15 \mathrm{~cm}$, mean 1/w 2, base rounded, apex acute, green, hairy; hair base broad, apex acute, transparent, $0.1 \mathrm{~cm}$ long. Lower glume absent. Upper glume ovate, $0.10 \times 0.05 \mathrm{~cm}$, mean 1/w 2, base truncate, apex acuminate, margins light green, membranous, hairy; hairs as on spikelet, pentanerved; nerves green, visible, glabrous. Lower lemma ovate, $0.20 \times 0.10 \mathrm{~cm}$, mean 1/w 2, base truncate, apex acute, margins entire, green, membranous, hairy; hairs as on spikelet, pentanerved; nerves green, visible, glabrous. Upper lemma ovate, $0.20 \mathrm{x} 0.10 \mathrm{~cm}$, mean 1/w 2, base truncate, apex acuminate, margins entire, green, coriaceous, pentanerved; nerves green, visible, glabrous. Upper palea elliptical $0.15 \times 0.10 \mathrm{~cm}$, mean 1/w 1.5, base truncate, apex denticulate, hyaline, glabrous, binerved; nerves not distinct, glabrous. Lodicules absent. Stamenes 3; anthers dithecous, versatile, dorsifixed, oblong, glabrous, yellow, base and apex rounded, $0.10 \mathrm{~cm}$ long. Ovary ovate, glabrous, translucent, $0.1 \mathrm{~cm}$ long; styles 2, linear, terete, glabrous, $0.05 \mathrm{~cm}$ long; stigma light brown, hairy; hair base broad, apex acute, in two row, $0.1 \mathrm{~cm}$ long. Caryopsis oblong, $0.15 \times 0.10 \mathrm{~cm}$, mean 1/w 1.5 , base and apex obtuse, purple, glabrous.

Seedling mesogeal. Primary root fibrous, branched, dull white, terete, $1.20 \mathrm{~cm}$ long at coleoptile stage and $1.6 \mathrm{~cm}$, $2.0, \mathrm{~cm}$ and $2.5 \mathrm{~cm}$ long at $1^{\mathrm{ST}}, 2^{\text {nd }}$, and $3^{\text {rd }}$ leaf stages respectively. Collet distinct, dull white and hairy; hair base broad, apex acute and $0.1 \mathrm{~cm}$ long. Scutellum oblong, $0.25 \times 0.10 \mathrm{~cm}$, mean 1/w 2.5, base and apex acute, dull white, slightly swollen and wavy. Mesocotyl light green, glabrous, terete and $0.4 \mathrm{~cm}$ long. Coleoptile oblong, $0.70 \times 0.25$ $\mathrm{cm}$, mean 1/w 2.8, base truncate, apex obtuse, compressed, light green, hyaline, glabrous and binerved. Adventitious roots appear after first leaf stage. First leaf sheath green, membranous, oblong, compressed, margins hyaline, and hairy ; hairs as on lemma. Ligule appears after $1^{\text {st }}$ leaf stage. First leaf blade elliptical, 0.80 x $0.25 \mathrm{~cm}$, mean 1/w 3.2, base truncate, apex obtuse, margins denticulate, green, membranous, hairy ; hairs as on leaf sheath; veins-5. Second leaf sheath same as that of first leaf sheath. Ligule membranous, glabrous and entire. Second leaf blade lanceolate, $1.0 \times 0.22 \mathrm{~cm}$, mean 1/w 5, base truncate, apex acute, margins denticulate, green, scabrid with 5 veins.

Flowering \& Fruiting:- September-April

Distribution: India, Tropical Asia and New Zealand

\section{Discussion:-}

It is evident from the keys provided that six congeneric taxa belonging to the genus Digiteria can be differentiated on the basis of node and leaf blade surface, ligule and prophyllum shape, number of racemes, rachis and pedicel shape, venation of glume and colour and shape of caryopsis. Similarly some interesting morphological features of seedlings are also significant systematically. Seedlings of reported can be differentiated and identified on the basis of ligule, lemma, collet, coleoptile and Scutellum. 


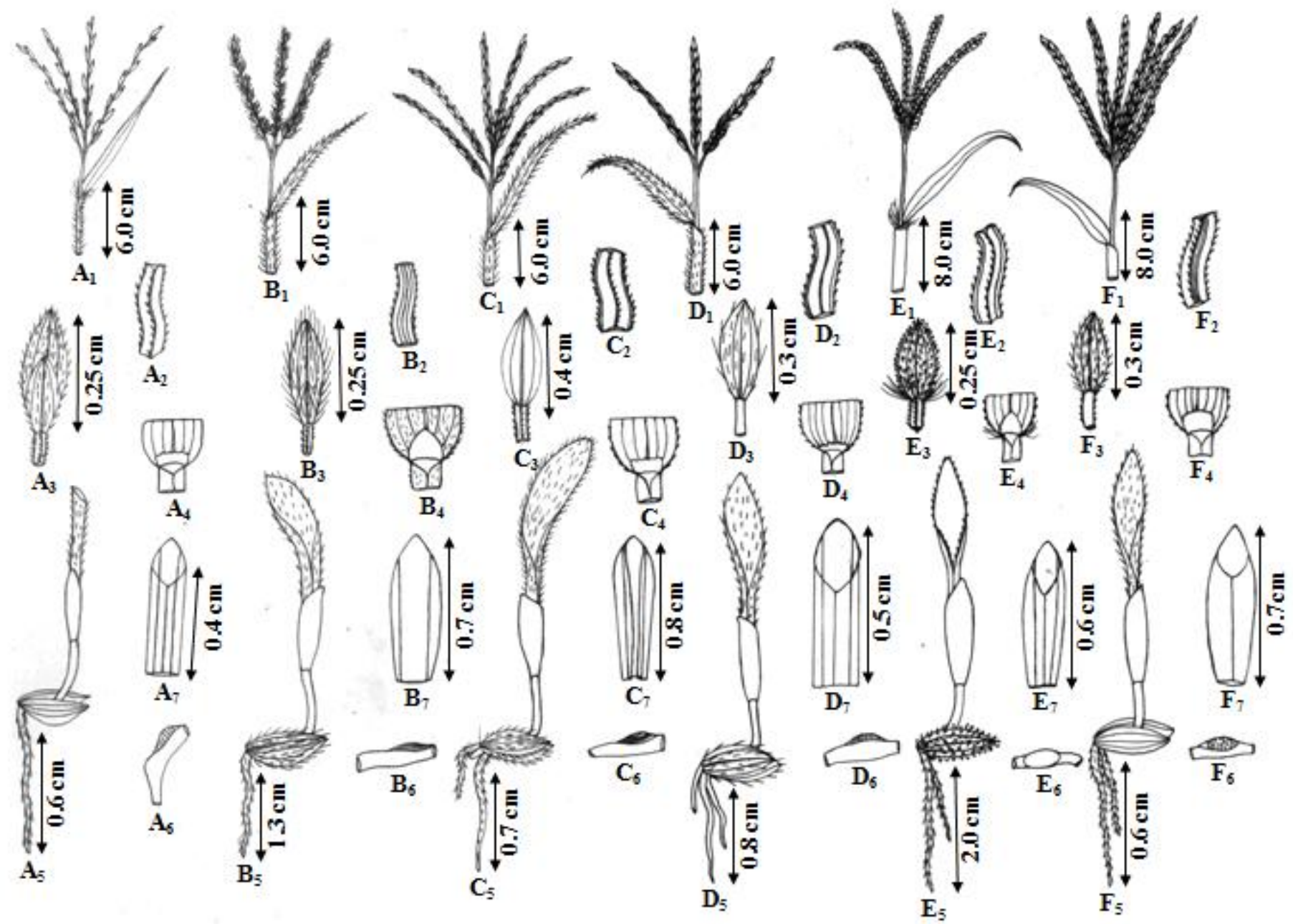

Figure 1:- A. D. abludens- $\mathbf{A}_{1}$. A twig, $\mathbf{A}_{2}$. Rachis, $\mathbf{A}_{3}$. Spikelet, $\mathbf{A}_{4}$. Ligule, $\mathbf{A}_{5}$. Seedling, $\mathbf{A}_{6}$. Scutellum, $\mathbf{A}_{7}$. Coleoptile; B. D.ciliaris- $\mathbf{B}_{1}$. A twig, $\mathbf{B}_{2}$. Rachis, $\mathbf{B}_{3}$. Spikelet, $\mathbf{B}_{4}$. Ligule, $\mathbf{B}_{5}$. Seedling, $\mathbf{B}_{6}$. Scutellum, $\mathbf{B}_{7}$. Coleoptile; C. D. griffithii- $\mathbf{C}_{1}$. A twig, $\mathbf{C}_{2}$. Rachis, $\mathbf{C}_{3}$. Spikelet, $\mathbf{C}_{4}$. Ligule, $\mathbf{C}_{5}$. Seedling, $\mathbf{C}_{6}$. Scutellum, $_{\mathbf{7}}$. Coleoptile; D. D. longiflora- $\mathbf{D}_{\mathbf{1}}$. A twig, $\mathbf{D}_{2}$. Rachis, $\mathbf{D}_{3}$. Spikelet, $\mathbf{D}_{\mathbf{4}}$. Ligule, $\mathbf{D}_{5}$. Seedling, $\mathbf{D}_{6}$. Scutellum, $\mathbf{D}_{7}$. Coleoptile; E. D. tarnata- $\mathbf{E}_{1}$. A twig, $\mathbf{E}_{2}$. Rachis, $\mathbf{E}_{3}$. Spikelet, $\mathbf{E}_{4}$. Ligule, $\mathbf{E}_{5}$. Seedling, $\mathbf{E}_{6}$. Scutellum, $\mathbf{E}_{7}$. Coleoptile; F. D. violascens- $\mathbf{F}_{1}$. A twig, $\mathbf{F}_{2}$. Rachis, $\mathbf{F}_{3}$. Spikelet, $\mathbf{F}_{4}$. Ligule, $\mathbf{F}_{5}$. Seedling, $\mathbf{F}_{6}$. Scutellum, $\mathbf{F}_{7}$. Coleoptile.

\section{Acknowledgements:-}

We are thankful to the Principal, Udai Pratap College (Autonomous), Varanasi, India for encouragement and UGC, New Delhi for financial support.

\section{References:-}

1. Clayton, W. D. and Renvoize, S. A. (1986): Genera Graminum. Grasses of the world. Kew Bulletin, Additional Series 13: 1-389.

2. De Wet, J.M.J. (1995): Minor Cereals. In: Smartt J, Simmonds NW (eds) Evolution of Crop Plants. (Longman: Burnt Mill), pp. 202-208.

3. Grass Phylogeny Working Group (GPWG) (2001):. Phylogeny and subfamilial classification of the grasses (Poaceae). Ann. Missouri Bot. Gard., 88: 373-457.

4. Haller, A.V. (1768): Historia Stirprum Indigenarum. Helvetae Inchoata, 2: 244. 
5. Quattrocchi, U. (2006): CRC world dictionary of grasses: common names, scientific names, eponyms, synonyms, and etymology. Volume 1, A - D. CRC Press (Taylor \& Francis Group), Boca Raton, FL.

6. Henrard, J.T. (1950): Monograph of the genus Digitaria. Leiden University, Netherland.

7. Heister, L. (1748): Systema plantarum generale ex fructificatione.

8. Holm, L.G., Plucknett, D.L., Pancho, J.V. and Herberger, J.P. (1977): The world's worst weeds: Distribution and biology. East-West Cent., Univ. Press Hawaii, Honolulu, HI. pp. 609.

9. Kellogg, E.A. (2006): Beyond taxonomy: Propects for understanding morphological diversity in the grasses (Poaceae). Darwiniana, 44: 7-17.

10. Morrone, O., Aagesen, L., Scataglini, M.A., Salariato, D.L., Denham, S.S., Chemisquy, M.A., Sede, S.M., Giussani, L.M., Kellogg, E.A. and F.O. Zuloaga (2012): Phylogeny of the Paniceae (Poaceae: Panicoideae): Integrating plastid DNA sequences and morphology into a new classification. Cladistics, 28:333-356.

11. Pozzobon, M.T., Glatzle, A., Conterato, I.F., Maria Teresa Schifino-Wittmann, M.T., and Grudsinske Smiderle, V.G. (2006): Variation in chromosome number and its relationship with agronomic characteristics in a germplasm collection of Digitaria eriantha sensu lato. Tropical Grasslands, 40: 157-164.

12. Singh, A. K., Sahu, R. K. and Singh, A. K. (2013): Seedling morphology of some Indian species of Eragrostis Wolf. (Poaceae). J. Econ. Taxon. Bot., 37(3): 576-581.

13. Vega, A., Rua, G., Fabbri, L., and Rugolo de Agrasar, Z. (2009): A morphology-based cladistic analysis of Digitaria (Poaceae, Panicoideae, Paniceae), Systematic Botany. 34: 312-323.

14. Watson, L. and Dallwitz, M.J. (1992): Grass Genera of the World: Descriptions, Illustrations, Identification, and Information Retrieval; including Synonyms, Morphology, Anatomy, Physiology, Phytochemistry, Cytology, Classification, Pathogens, World and Local Distribution, and References. http://biodiversity.uno.edu/delta/

15. Zuloaga, F.O., Morrone, O., Rúgolo De Agrasar, Z.E., Anton, A.M., Arriaga, M.O., and Cialdella, A.M. (1994): Gramineas V. In: Spichiger R, Ramella L (eds) Flora del Paraguay. Vol. 23. (Conservatoire et Jardin Botanique: Ville de Genève). 